\title{
Are beryllium abundances anomalous in stars with giant planets?^
}

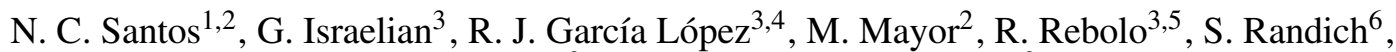 \\ A. Ecuvillon ${ }^{3}$, and C. Domínguez Cerdeña ${ }^{3}$ \\ ${ }^{1}$ Centro de Astronomia e Astrofísica da Universidade de Lisboa, Observatório Astronómico de Lisboa, Tapada da Ajuda, \\ 1349-018 Lisboa, Portugal \\ e-mail: Nuno.Santos@oal.ul.pt \\ 2 Observatoire de Genève, 51 Ch. des Maillettes, 1290 Sauverny, Switzerland \\ 3 Instituto de Astrofísica de Canarias, 38200 La Laguna, Tenerife, Spain \\ ${ }^{4}$ Departamento de Astrofísica, Universidad de La Laguna, Av. Astrofísico Francisco Sánchez s/n, 38206, La Laguna, \\ Tenerife, Spain \\ 5 Consejo Superior de Investigaciones Científicas, Spain \\ 6 INAF/Osservatorio Astrofisico di Arcetri, Largo Fermi 5, 50125 Firenze, Italy
}

Received 24 March 2004 / Accepted 22 July 2004

\begin{abstract}
In this paper we present beryllium (Be) abundances in a large sample of 41 extra-solar planet host stars, and for 29 stars without any known planetary-mass companion, spanning a large range of effective temperatures. The Be abundances were derived through spectral synthesis done in standard Local Thermodynamic Equilibrium, using spectra obtained with various instruments. The results seem to confirm that overall, planet-host stars have "normal" Be abundances, although a small, but not significant, difference might be present. This result is discussed, and we show that this difference is probably not due to any stellar "pollution" events. In other words, our results support the idea that the high-metal content of planet-host stars has, overall, a "primordial" origin. However, we also find a small subset of planet-host late-F and early-G dwarfs that might have higher than average Be abundances. The reason for the offset is not clear, and might be related either to the engulfment of planetary material, to galactic chemical evolution effects, or to stellar-mass differences for stars of similar temperature.
\end{abstract}

Key words. stars: abundances - stars: fundamental parameters - stars: planetary systems stars: planetary systems: formation - stars: atmospheres

\section{Introduction}

The study of the chemical abundances in planet-host stars (e.g. Gonzalez 1998; Santos et al. 2001; Gonzalez et al. 2001; Reid 2002; Santos et al. 2003b; Laws et al. 2003; Israelian 2003; Santos et al. 2004a) has revealed the important role that the global metallicity plays in the formation of giant planets. It has been shown that the probability of finding a planet is a steeply rising function of the metal content of the star (e.g. Santos et al. 2001; Reid 2002; Santos et al. 2003b, 2004a). This crucial observation is helping us to better understand the mechanisms involved in the formation of the planetary systems (for a general review on extra-solar planets see e.g. Santos et al. 2003a).

^ Based on observations collected with the VLT/UT2 Kueyen telescope (Paranal Observatory, ESO, Chile) using the UVES spectrograph (Observing runs 66.C-0116 A, 66.D-0284 A, and 68.C-0058 A), and with the William Herschel and Nordic Optical Telescopes, operated on the island of La Palma by the Isaac Newton Group and jointly by Denmark, Finland, Iceland, and Norway, respectively, in the Spanish Observatorio del Roque de los Muchachos of the Instituto de Astrofísica de Canarias.
Although most studies of the chemical abundances of stars hosting giant planets have concentrated on the analysis of iron and other iron-peak elements, alpha-elements, and other metals (e.g. Santos et al. 2000; Gonzalez \& Laws 2000; Smith et al. 2001; Sadakane et al. 2002; Bodaghee et al. 2003; Ecuvillon et al. 2004; Santos et al. 2004a), a few have also explored the abundances of the light elements ${ }^{6} \mathrm{Li}$ (Israelian et al. 2001; Reddy et al. 2003; Israelian et al. 2003), ${ }^{7} \mathrm{Li}$ (Gonzalez \& Laws 2000; Ryan 2000; Israelian et al. 2004), and ${ }^{9} \mathrm{Be}$ (García López \& Pérez de Taoro 1998; Deliyannis et al. 2000; Santos et al. 2002a). Overall, and putting aside a few exceptions (e.g. Israelian et al. 2001; Laws \& Gonzalez 2001), these studies suggest that stars with planets have in general normal light-element abundances, typical of field stars, even though a few interesting correlations have been found (e.g. Israelian et al. 2004).

The study of the light elements has an enormous potential for the understanding of planet formation. First, it is well known that light elements and their abundance ratios are good tracers of stellar internal mixing and rotation 
Table 1. Spectrographs used for the current study, their spectral resolution, and date of the observations.

\begin{tabular}{lccc}
\hline \hline Spectrograph/Telescope & $\begin{array}{c}\text { Resolution } \\
(\lambda / \Delta \lambda)\end{array}$ & $\begin{array}{c}\text { Date of } \\
\text { observations }\end{array}$ & Designation \\
\hline UES/4.2-m William Herschel Telescope & 55000 & Aug. 1998 & UES \\
IACUB/2.6-m Nordic Optical Telescope & 35000 & May 2000 & IACUB(A) \\
UVES/VLT 8.2-m Kueyen UT2 (VLT) & 70000 & Nov. 2000-Jan. 2001 & UVES(A) \\
UVES/VLT 8.2-m Kueyen UT2 (VLT) & 56000 & Feb. 2001 & UVES(B) \\
IACUB/2.6-m Nordic Optical Telescope & 35000 & Oct. 2001 & IACUB(B) \\
UVES/VLT 8.2-m Kueyen UT2 (VLT) & 70000 & Oct. 2001-Mar. 2002 & UVES(C) \\
\hline
\end{tabular}

(e.g. Pinsonneault et al. 1990; Stephens et al. 1997). From the several mixing mechanisms that have been referred to in the literature as responsible for the depletion of light-elements in solar-type stars, rotation and angular momentum loss are among the leading processes (see discussion in Stephens et al. 1997). The study of light element abundances may thus probably tell us much about processes related to the angular momentum evolution of planet-host stars. If the formation of giant planets needs the presence of massive proto-planetary disks, we can eventually expect that planet hosts and "single" stars might have had a different angular momentum history, thus presenting different light-element abundances. Although not completely established, a relation between the disk mass and the rotation history of a star might indeed exist (e.g. Edwards et al. 1993; Strom 1994; Stassun et al. 1999; Barnes et al. 2001; Rebull 2001, 2002; Hartmann 2002; Wolff et al. 2004). There is even some theoretical evidence suggesting that disk life-times might be related to the formation and presence of planets (Goodman \& Rafikov 2001; Sari \& Goldreich 2004). Finally, an angularmomentum variation can also be induced by the eventual accretion of planetary-mass bodies into the star (e.g. Siess \& Livio 1999; Israelian et al. 2003).

But other mechanisms exist capable of inducing differences in the light-element abundances between planet-host stars and stars without planets. In particular, if at least part of the metal "enrichment" found for planet host stars is due to stellar pollution effects (e.g. Laughlin \& Adams 1997; Murray \& Chaboyer 2002; Vauclair 2004), we should also be able to observe an enhancement in the abundances of the light elements in planet hosts (e.g. Israelian et al. 2001; Pinsonneault et al. 2001; Santos et al. 2002a; Israelian et al. 2003). This enhancement should be at least of the same order of magnitude as the excess metallicity observed, although subsequent Li and Be depletion could mask the "pollution" effect.

In this paper we continue our study of beryllium (Be) abundances already started in Santos et al. (2002a), by increasing the number of stars in our samples with new data taken with the UVES spectrograph (at the VLT/UT2 Kueyen telescope). The Be abundances are further compared with $\mathrm{Li}$ abundances of the same targets. The analysis reveals that overall, and except for a few cases, there are no clear differences between planet hosts and stars without any known planetary companion. The implications of this result are discussed. Our analysis also suggests that Be depletion for stars of different effective temperatures does not behave as expected from models, and that there seems to exist a Be-gap for solar-temperature stars. These results are discussed in a separate paper (Santos et al. 2004b) hereafter, Paper II.

\section{The data}

Part of the spectra analyzed in this paper has already been used in Santos et al. (2002a). Meanwhile, however, we have gathered near-UV spectra for more targets using the UVES spectrograph at the 8.2-m Kueyen VLT (UT2) telescope (run ID 68.C-0058 A). These new spectra have a spectral resolution $R \sim 70000$, and $S / N$ ratios usually between 100 and 200. For a more complete description of the data obtained with other instruments used we refer to Santos et al. (2002a).

In Table 1 we describe the instruments and telescopes used to obtain our data, as well as the spectral resolution and the date of the observations. In Tables 2 and 3 we list the instrument used to observe each star as well as the signal-to-noise achieved.

All the data were reduced using IRAF $^{1}$ tools in the echelle package. Standard background correction, flat-field, and extraction procedures were used. For the UVES(A), UVES(C), and UES runs the wavelength calibration was done using a ThAr lamp spectrum taken during the same night. For the UVES(B), IACUB(A), and IACUB(B) runs the wavelength calibration was done using photospheric lines in the region of interest.

\section{Be abundances}

\subsection{Stellar parameters}

The stellar atmospheric parameters for most of our targets were taken from Santos et al. (2004a), who have obtained accurate and uniform stellar parameters for 98 extra-solar planet-host stars, as well as for our comparison sample of objects. The errors in the different stellar parameters are of the order of $50 \mathrm{~K}$ in $T_{\text {eff }}, 0.12 \mathrm{dex}$ in $\log g, 0.10 \mathrm{~km} \mathrm{~s}^{-1}$ in the microturbulence parameter, and $0.05 \mathrm{dex}$ in $[\mathrm{Fe} / \mathrm{H}])$. Only for BD -103166 were the stellar parameters taken from Gonzalez et al. (2001), who have used a similar technique to derive them. This procedure

${ }^{1}$ IRAF is distributed by National Optical Astronomy Observatories, operated by the Association of Universities for Research in Astronomy, Inc., under contract with the National Science Foundation, USA. 
Table 2. Derived Be abundances for the planet hosts stars in our study.

\begin{tabular}{|c|c|c|c|c|c|c|c|c|c|c|c|}
\hline 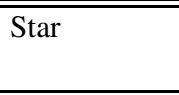 & $\begin{array}{l}T_{\text {eff }} \\
{[\mathrm{K}]} \\
\end{array}$ & $\begin{array}{l}\log g_{\text {spec }} \\
{\left[\mathrm{cm} \mathrm{s}^{-2}\right]}\end{array}$ & $\begin{array}{c}\xi_{t} \\
{\left[\mathrm{~km} \mathrm{~s}^{-1}\right]}\end{array}$ & $\overline{[\mathrm{Fe} / \mathrm{H}]}$ & $\overline{l o g} N(\mathrm{Be})$ & $\sigma(\mathrm{Be})$ & $\overline{\text { Instr. }^{\dagger}}$ & $S / N$ & $\begin{array}{c}v \sin i \\
{\left[\mathrm{~km} \mathrm{~s}^{-1}\right]}\end{array}$ & source $^{\dagger \dagger}$ & $\overline{\log N(\mathrm{Li})}$ \\
\hline BD -103166 & 5320 & 4.38 & 0.85 & 0.33 & $<0.50$ & - & [2] & 20 & 1.58 & (a) & - \\
\hline HD 6434 & 5835 & 4.60 & 1.53 & -0.52 & 1.08 & 0.10 & [3] & 150 & 1.30 & (a) & $<0.8$ \\
\hline HD 9826 & 6212 & 4.26 & 1.69 & 0.13 & 1.05 & 0.13 & [6] & 120 & 9 & (b) & 2.55 \\
\hline HD 10647 & 6143 & 4.48 & 1.40 & -0.03 & 1.19 & 0.10 & [3] & 150 & 4.87 & (a) & 2.80 \\
\hline HD 10697 & 5641 & 4.05 & 1.13 & 0.14 & 1.31 & 0.13 & [5] & 40 & - & - & 1.96 \\
\hline HD 12661 & 5702 & 4.33 & 1.05 & 0.36 & 1.13 & 0.13 & [5] & 40 & - & - & $<0.98$ \\
\hline HD 13445 & 5163 & 4.52 & 0.72 & -0.24 & $<0.40$ & - & [1] & 150 & 1.27 & (a) & $<-0.12$ \\
\hline HD 16141 & 5801 & 4.22 & 1.34 & 0.15 & 1.17 & 0.13 & [1] & 120 & 1.95 & (a) & 1.11 \\
\hline HD 17051 & 6252 & 4.61 & 1.18 & 0.26 & 1.03 & 0.13 & [1] & 150 & 5.38 & (a) & 2.66 \\
\hline HD 19994 & 6190 & 4.19 & 1.54 & 0.24 & 0.93 & 0.12 & [3] & 140 & 8.10 & (a) & 1.99 \\
\hline HD 22049 & 5073 & 4.43 & 1.05 & -0.13 & 0.80 & 0.13 & [5] & 100 & 2.13 & (a) & $<0.25$ \\
\hline HD 22049 & 5073 & 4.43 & 1.05 & -0.13 & 0.75 & 0.31 & [3] & 200 & 2.13 & (a) & $<0.25$ \\
\hline HD 22049 & 5073 & 4.43 & 1.05 & -0.13 & 0.77 & - & avg & - & 2.13 & (a) & $<0.25$ \\
\hline HD 27442 & 4825 & 3.55 & 1.18 & 0.39 & $<0.30$ & - & [3] & 110 & 0 & (a) & $<-0.47$ \\
\hline HD 38529 & 5674 & 3.94 & 1.38 & 0.40 & $<-0.10$ & - & [2] & 60 & - & - & $<0.61$ \\
\hline HD 46375 & 5268 & 4.41 & 0.97 & 0.20 & $<0.80$ & - & [3] & 90 & - & - & $<-0.02$ \\
\hline HD 52265 & 6103 & 4.28 & 1.36 & 0.23 & 1.25 & 0.11 & [1] & 120 & 3.95 & (a) & 2.88 \\
\hline HD 75289 & 6143 & 4.42 & 1.53 & 0.28 & 1.38 & 0.10 & [2] & 30 & 3.81 & (a) & 2.85 \\
\hline HD 75289 & 6143 & 4.42 & 1.53 & 0.28 & 1.33 & 0.12 & [1] & 110 & 3.81 & (a) & 2.85 \\
\hline HD 75289 & 6143 & 4.42 & 1.53 & 0.28 & 1.36 & - & avg & 110 & 3.81 & (a) & 2.85 \\
\hline HD 82943 & 6016 & 4.46 & 1.13 & 0.30 & 1.37 & 0.17 & [4] & 20 & 1.65 & (a) & 2.51 \\
\hline HD 82943 & 6016 & 4.46 & 1.13 & 0.30 & 1.27 & 0.12 & [2] & 35 & 1.65 & (a) & 2.51 \\
\hline HD 82943 & 6016 & 4.46 & 1.13 & 0.30 & 1.27 & 0.12 & [1] & 140 & 1.65 & (a) & 2.51 \\
\hline HD $82943^{\star}$ & 6016 & 4.46 & 1.13 & 0.30 & 1.27 & - & avg & 140 & 1.65 & (a) & 2.51 \\
\hline HD 83443 & 5454 & 4.33 & 1.08 & 0.35 & $<0.70$ & - & [3] & 100 & 1.38 & (a) & $<0.52$ \\
\hline HD 92788 & 5821 & 4.45 & 1.16 & 0.32 & 1.19 & 0.11 & [2] & 40 & 1.78 & (a) & 1.34 \\
\hline HD 95128 & 5954 & 4.44 & 1.30 & 0.06 & 1.23 & 0.11 & [4] & 100 & 2.1 & (c) & 1.83 \\
\hline HD 108147 & 6248 & 4.49 & 1.35 & 0.20 & 0.99 & 0.10 & {$[2]$} & 60 & 5.34 & (a) & 2.33 \\
\hline HD 114762 & 5884 & 4.22 & 1.31 & -0.70 & 0.82 & 0.11 & [4] & 65 & 1.5 & (c) & 2.20 \\
\hline HD 117176 & 5560 & 4.07 & 1.18 & -0.06 & 0.86 & 0.13 & [4] & 70 & 0.5 & (c) & 1.88 \\
\hline HD 120136 & 6339 & 4.19 & 1.70 & 0.23 & $<0.25$ & - & [6] & 90 & 14.5 & (b) & - \\
\hline HD 121504 & 6075 & 4.64 & 1.31 & 0.16 & 1.33 & 0.11 & [2] & 45 & 2.56 & (a) & 2.65 \\
\hline HD 130322 & 5392 & 4.48 & 0.85 & 0.03 & 0.95 & 0.13 & [4] & 35 & 1.47 & (a) & $<0.13$ \\
\hline HD 134987 & 5776 & 4.36 & 1.09 & 0.30 & 1.22 & 0.11 & [2] & 60 & 2.22 & (a) & $<0.74$ \\
\hline HD 143761 & 5853 & 4.41 & 1.35 & -0.21 & 1.11 & 0.12 & [6] & 120 & 1.5 & (c) & 1.46 \\
\hline HD 145675 & 5311 & 4.42 & 0.92 & 0.43 & $<0.65$ & - & [4] & 65 & 1 & (d) & $<0.03$ \\
\hline HD 168443 & 5617 & 4.22 & 1.21 & 0.06 & 1.11 & 0.13 & [4] & 55 & 1.68 & (a) & $<0.78$ \\
\hline HD 169830 & 6299 & 4.10 & 1.42 & 0.21 & $<-0.40$ & - & [3] & 130 & 3.35 & (a) & $<1.16$ \\
\hline HD 179949 & 6260 & 4.43 & 1.41 & 0.22 & 1.08 & 0.10 & [3] & 100 & 6.10 & (a) & 2.65 \\
\hline HD 187123 & 5845 & 4.42 & 1.10 & 0.13 & 1.08 & 0.12 & [4] & 55 & 1.73 & (d) & 1.21 \\
\hline HD 192263 & 4947 & 4.51 & 0.86 & -0.02 & $<0.90$ & - & [3] & 60 & 2.02 & (a) & $<-0.39$ \\
\hline HD 195019 & 5842 & 4.32 & 1.27 & 0.08 & 1.15 & 0.12 & [4] & 50 & 1.73 & (a) & 1.47 \\
\hline HD 202206 & 5752 & 4.50 & 1.01 & 0.35 & 1.04 & 0.11 & [3] & 130 & 2.44 & (a) & 1.04 \\
\hline HD 209458 & 6117 & 4.48 & 1.40 & 0.02 & 1.24 & 0.11 & [3] & 150 & 3.65 & (a) & 2.70 \\
\hline HD 210277 & 5532 & 4.29 & 1.04 & 0.19 & 0.91 & 0.13 & [1] & 110 & 1.39 & (a) & $<0.30$ \\
\hline HD 217014 & 5804 & 4.42 & 1.20 & 0.20 & 1.02 & 0.12 & [6] & 100 & 2.1 & (c) & 1.30 \\
\hline HD 217107 & 5646 & 4.31 & 1.06 & 0.37 & 0.96 & 0.13 & [1] & 120 & 1.37 & (a) & $<0.40$ \\
\hline HD 222582 & 5843 & 4.45 & 1.03 & 0.05 & 1.14 & 0.11 & [3] & 125 & 1.75 & (a) & $<0.59$ \\
\hline
\end{tabular}

$\dagger$ The instruments used to obtain the spectra were: [1] UVES(A); [2] UVES(B); [3] UVES(C); [4] IACUB(A); [5] IACUB(B); [6] UES.

${ }^{\dagger}$ The sources of the $v \sin i$ are: (a) CORALIE (Santos et al. 2002b), (b) Gonzalez (1997), (c) Gonzalez (1998), (d) Naef et al. (2004).

* Given the lower $S / N$ of the IACUB spectrum, only the UVES spectra were considered. 
Table 3. Derived Be abundances for the comparison stars in our study.

\begin{tabular}{|c|c|c|c|c|c|c|c|c|c|c|c|}
\hline Star & $\begin{array}{l}T_{\text {eff }} \\
{[\mathrm{K}]} \\
\end{array}$ & $\begin{array}{l}\log g_{\text {spec }} \\
{\left[\mathrm{cm} \mathrm{s}^{-2}\right]}\end{array}$ & $\begin{array}{c}\xi_{t} \\
{\left[\mathrm{~km} \mathrm{~s}^{-1}\right]}\end{array}$ & $\bar{~}[\mathrm{Fe} / \mathrm{H}]$ & $\log N(\mathrm{Be})$ & $\sigma(\mathrm{Be})$ & Instr. $^{\dagger}$ & $S / N$ & $\begin{array}{c}v \sin i \\
{\left[\mathrm{~km} \mathrm{~s}^{-1}\right]}\end{array}$ & source $^{\dagger \dagger}$ & $\log N(\mathrm{Li})$ \\
\hline HD 870 & 5447 & 4.57 & 1.13 & -0.07 & 0.80 & 0.15 & [1] & 130 & 1.77 & (a) & $<0.20$ \\
\hline HD 1461 & 5768 & 4.37 & 1.27 & 0.17 & 1.14 & 0.13 & [1] & 120 & 1.71 & (a) & $<0.51$ \\
\hline HD 1581 & 5956 & 4.39 & 1.07 & -0.14 & 1.15 & 0.11 & [1] & 140 & 2.16 & (a) & 2.37 \\
\hline HD 3823 & 5948 & 4.06 & 1.17 & -0.25 & 1.02 & 0.12 & [1] & 130 & 1.99 & (a) & 2.41 \\
\hline HD 4391 & 5878 & 4.74 & 1.13 & -0.03 & 0.64 & 0.11 & [3] & 150 & 2.72 & (a) & $<1.09$ \\
\hline HD 7570 & 6140 & 4.39 & 1.50 & 0.18 & 1.17 & 0.10 & [3] & 180 & 3.82 & (a) & 2.91 \\
\hline HD 10700 & 5344 & 4.57 & 0.91 & -0.52 & 0.83 & 0.11 & [3] & 180 & 0.90 & (a) & $<0.41$ \\
\hline HD 14412 & 5368 & 4.55 & 0.88 & -0.47 & 0.80 & 0.11 & [3] & 190 & 1.42 & (a) & $<0.44$ \\
\hline HD 20010 & 6275 & 4.40 & 2.41 & -0.19 & 1.01 & 0.10 & [3] & 180 & 4.63 & (a) & 2.13 \\
\hline HD 20766 & 5733 & 4.55 & 1.09 & -0.21 & $<-0.09$ & - & [3] & 200 & 1.98 & (a) & $<0.97$ \\
\hline HD 20794 & 5444 & 4.47 & 0.98 & -0.38 & 0.91 & 0.11 & [3] & 250 & 0.52 & (a) & $<0.52$ \\
\hline HD 20807 & 5843 & 4.47 & 1.17 & -0.23 & 0.36 & 0.11 & [3] & 160 & 1.74 & (a) & $<1.07$ \\
\hline HD 23249 & 5074 & 3.77 & 1.08 & 0.13 & $<0.15$ & - & [5] & 80 & 1.01 & (a) & 1.24 \\
\hline HD 23484 & 5176 & 4.41 & 1.03 & 0.06 & $<0.70$ & - & [3] & 140 & 2.40 & (a) & $<0.40$ \\
\hline HD 26965 A & 5126 & 4.51 & 0.60 & -0.31 & 0.76 & 0.13 & [5] & 55 & 0.77 & (a) & $<0.17$ \\
\hline HD 30495 & 5868 & 4.55 & 1.24 & 0.02 & 1.16 & 0.11 & [3] & 140 & 3.04 & (a) & 2.44 \\
\hline HD 36435 & 5479 & 4.61 & 1.12 & 0.00 & 0.99 & 0.12 & [3] & 210 & 4.58 & (a) & 1.67 \\
\hline HD 38858 & 5752 & 4.53 & 1.26 & -0.23 & 1.02 & 0.11 & [3] & 150 & 0.99 & (a) & 1.64 \\
\hline HD 43162 & 5633 & 4.48 & 1.24 & -0.01 & 1.08 & 0.11 & [3] & 160 & 5.49 & (a) & 2.34 \\
\hline HD 43834 & 5594 & 4.41 & 1.05 & 0.10 & 0.94 & 0.11 & [3] & 220 & 1.44 & (a) & 2.30 \\
\hline HD 69830 & 5410 & 4.38 & 0.89 & -0.03 & 0.79 & 0.11 & [3] & 100 & 0.75 & (a) & $<0.47$ \\
\hline HD 72673 & 5242 & 4.50 & 0.69 & -0.37 & 0.70 & 0.13 & [3] & 180 & 1.19 & (a) & $<0.48$ \\
\hline HD 74576 & 5000 & 4.55 & 1.07 & -0.03 & 0.70 & 0.31 & [3] & 120 & 3.56 & (a) & 1.72 \\
\hline HD 76151 & 5803 & 4.50 & 1.02 & 0.14 & 1.02 & 0.11 & [3] & 110 & 1.02 & (a) & 1.88 \\
\hline HD 84117 & 6167 & 4.35 & 1.42 & -0.03 & 1.11 & 0.11 & [3] & 160 & 4.85 & (a) & 2.64 \\
\hline HD 189567 & 5765 & 4.52 & 1.22 & -0.23 & 1.06 & 0.10 & [3] & 160 & 1.29 & (a) & $<0.82$ \\
\hline HD 192310 & 5069 & 4.38 & 0.79 & -0.01 & $<0.60$ & - & [3] & 180 & 0.85 & (a) & $<0.20$ \\
\hline HD 211415 & 5890 & 4.51 & 1.12 & -0.17 & 1.12 & 0.10 & [3] & 190 & 1.84 & (a) & 1.92 \\
\hline HD 222335 & 5260 & 4.45 & 0.92 & -0.16 & 0.66 & 0.22 & [1] & 110 & 1.25 & (a) & $<0.31$ \\
\hline
\end{tabular}

† The instruments used to obtain the spectra were: [1] UVES(A); [2] UVES(B); [3] UVES(C); [4] IACUB(A); [5] IACUB(B); [6] UES.

${ }^{\dagger \dagger}$ The sources of the $v \sin i$ are: (a) CORALIE (Santos et al. 2002b), (b) Gonzalez (1997), (c) Gonzalez (1998), (d) Naef et al. (2004).

gives us the guarantee that the current analysis has no systematic errors due to stellar parameter determination (e.g. due to different effective temperature scales).

For three of the stars (HD 870, HD 1461, and HD 3823 for which no planetary companions have been found to date), no parameters were available, as these stars were not part of the volume-limited comparison sample studied in Santos et al. (2001, 2004a). As already done in Santos et al. (2002a), these were derived from CORALIE or FEROS spectra, in the same way as for all the other stars.

\subsection{Spectral synthesis}

The abundance analysis was done in standard Local Thermodynamic Equilibrium (LTE) using the 2002 version of the code MOOG $^{2}$ (Sneden 1973), and a grid of Kurucz (1993) ATLAS9 atmospheres. Be abundances were derived by fitting synthetic spectra to the data, using the same line list as in García López \& Pérez de Taoro (1998). While both Be II lines

\footnotetext{
2 The source code of MOOG2002 can be downloaded at http://verdi.as.utexas.edu/moog.html
}

at 3130.420 and $3131.065 \AA$ are present in our data, we only used the latter, given the severe line blending in the region around $3130.420 \AA$ (which has been used only for checking the consistency of the fit).

We derived the Be abundance for the Sun using a Kurucz model atmosphere (Kurucz 1993) with $T_{\text {eff }}=5777 \mathrm{~K}$, $\log g=4.44$, and $\xi_{t}=1.0$, and fitting the Kurucz Solar spectrum (Kurucz et al. 1984), after degrading its resolution to 70000 , a value similar to the resolution of the UVES spectra used in this paper. We used a smoothing profile based on its $v \sin i\left(1.9 \mathrm{~km} \mathrm{~s}^{-1}\right.$ - Soderblom 1982), a macroturbulence of $3.0 \mathrm{~km} \mathrm{~s}^{-1}$, and a limb darkening coefficient of 0.6 . The derived $\mathrm{Be}$ abundance is $\log N(\mathrm{Be})=1.10$, only 0.05 dex below the value obtained by Chmielewski et al. (1975). The small difference found is probably due to the different solar model used by García López \& Pérez de Taoro (1998) to build the line-list. Since we are mostly interested in a relative comparison, no changes were made in the line-list, and a solar value of $\log N(\mathrm{Be})=1.10$ is considered in the rest of the paper.

When performing the spectral synthesis of the UES and UVES data, the synthetic spectra were convolved with a Gaussian smoothing profile and a radial-tangential profile to 


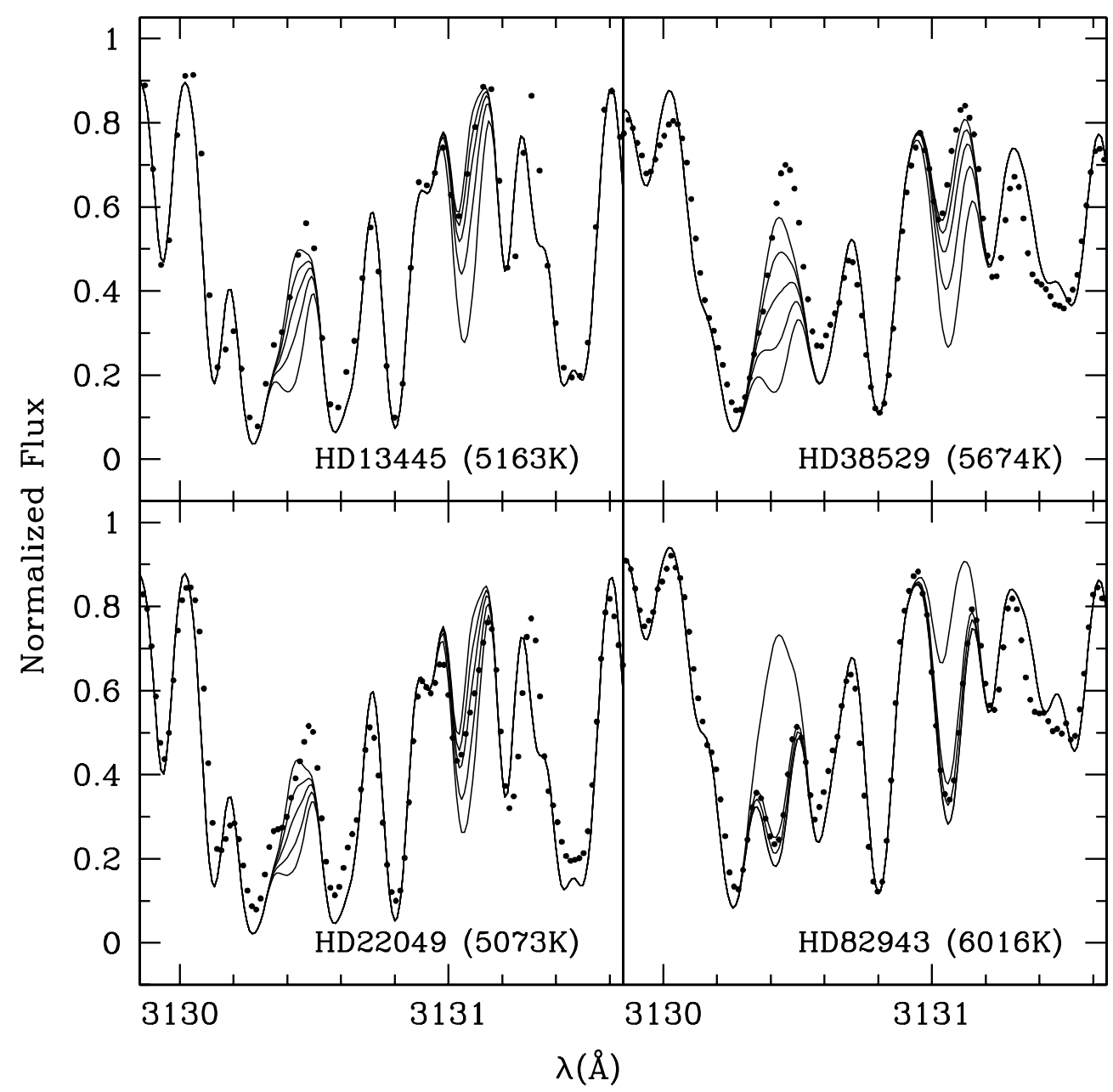

Fig. 1. Spectral syntheses (lines) and observed spectra (dots) in the Be II line region for 4 extra-solar planet-host stars of different effective temperatures (see Table 2). In all panels, the upper and lower syntheses were done with a $\log N(\mathrm{Be})$ of 1.42 (meteoritic) and -10.0 (essentially no Be). Lower panels: For HD 82943, the three other spectral syntheses correspond to the optimal fit and to fits with abundance variations of \pm 0.15 dex. For HD 22049, the three intermediate fits were done with Be abundances of 1.05, 0.75 and 0.25 dex. Upper panels: fits for two stars for which we have obtained only upper limits for the Be abundances. HD 13445: the syntheses correspond to Be abundances of 1.42, 0.90, 0.50, 0.10, and -10.0; HD 38529: the syntheses correspond to Be abundances of 1.42, 0.80, 0.40, -0.10 , and -10.0 . Stellar effective temperatures are also shown.

take into account the spectral resolution and the macroturbulence (this latter was varied between 1.0 and $5.0 \mathrm{~km} \mathrm{~s}^{-1}$, between K and F dwarfs - Gray 1992), respectively. A rotational profile was also added to account for the projected rotational velocity of the stars. The stellar $v \sin i$, listed in Tables 2 and 3, was for most of the cases estimated from the width of the CORALIE cross-correlation function (see Appendix of Santos et al. 2002b). This method gives excellent results, as illustrated by HD 82943, for which we have obtained the same value of $v \sin i=1.65 \mathrm{~km} \mathrm{~s}^{-1}$ using the CORALIE CCF and a detailed spectral synthesis method (Israelian et al. 2001). A limb darkening coefficient of 0.6 was considered for all cases, and the overall metallicity was scaled to the iron abundance.

For the IACUB data only a Gaussian smoothing profile was added, to take into account the instrumental profile. In this case, although the spectrograph can provide $R \sim 50000$ data, the observations were carried out at lower resolution to improve its efficiency in the Be II spectral region (as the instrument was attached to a telescope of $2.5-\mathrm{m}$ diameter). At this resolving power, the instrumental profile dominates the broadening of the observed spectra (note the low $v \sin i$ of the targets).

We then iterated by changing the Be abundance and the continuum placement until the best fit for the whole spectral region was obtained. In this procedure, the global fit in the wavelength interval from 3129.5 to $3132 \AA$ \&as considered, and not simply the region around the Be II lines. Small changes of the Gaussian smoothing profile were allowed when judged necessary (although these changes were always very small). The resulting abundances for all the objects observed are listed in Tables 2 and 3. Here we use the notation $\log N(\mathrm{Be})=[\mathrm{Be}]=$ $\log (\mathrm{Be} / \mathrm{H})+12$. Some examples of fits are shown in Figs. 1 and 2 (for more of such plots we refer to Paper II).

For HD 120136 ( $\tau$ Boo), the Be determination was particularly difficult since this $\mathrm{F}$ dwarf has a $v \sin i$ higher than all the other stars $\left(\sim 15 \mathrm{~km} \mathrm{~s}^{-1}\right)$. However, and as we can see from Fig. 3, from our spectral synthesis we can be sure that this star has already depleted a good part of its Be. Given the difficulty of the fit, however, the upper limit that we have determined for 


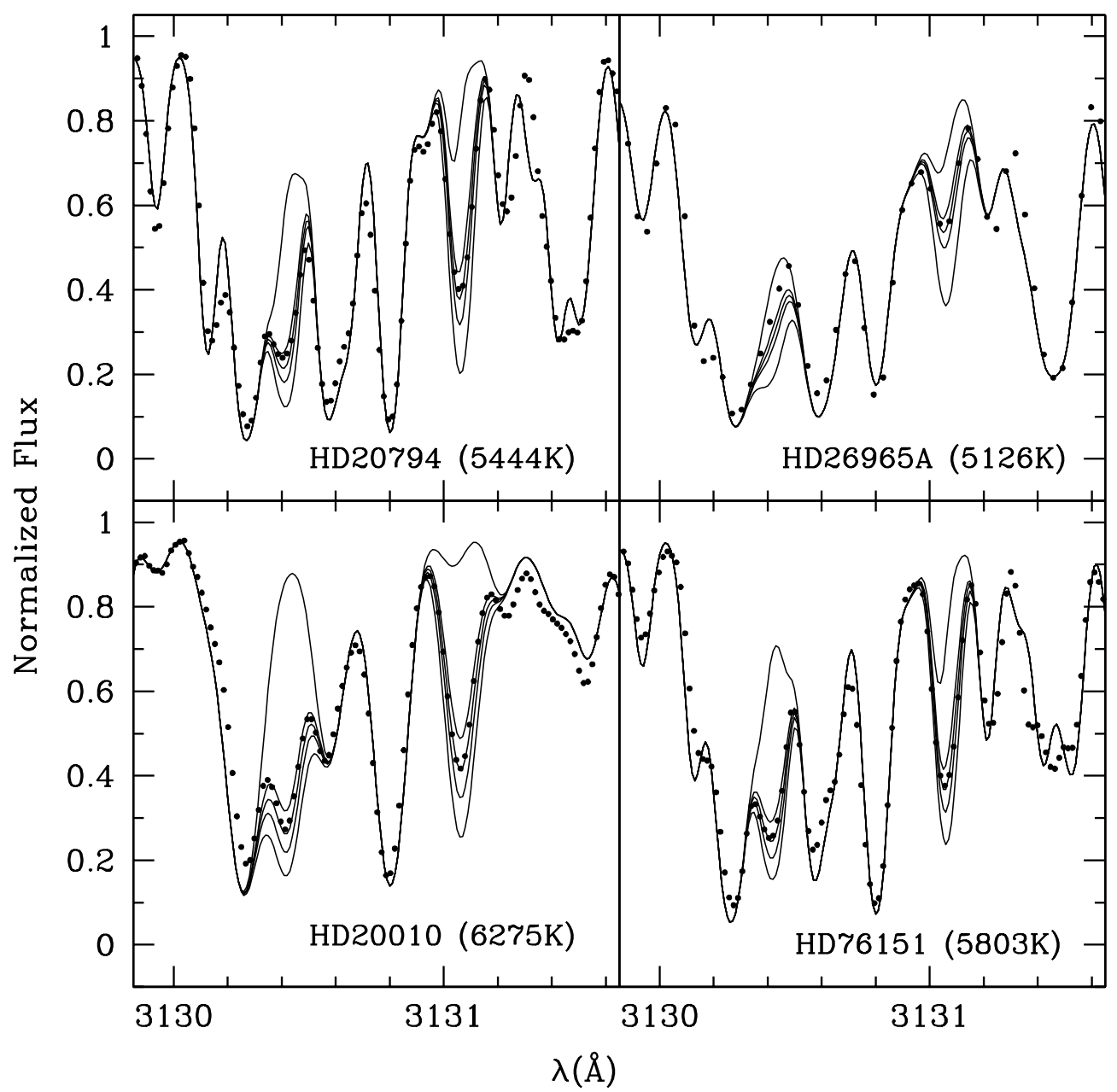

Fig. 2. Same as Fig. 1 but for 4 stars for which no planetary companions have been detected. In all panels we present the observed data and synthetic spectra obtained with meteoritic and "no-Be" (upper and lower fits, respectively), together with three other syntheses. These correspond to the optimal fit and to fits with abundance variations of \pm 0.15 dex. Stellar effective temperatures are also shown.

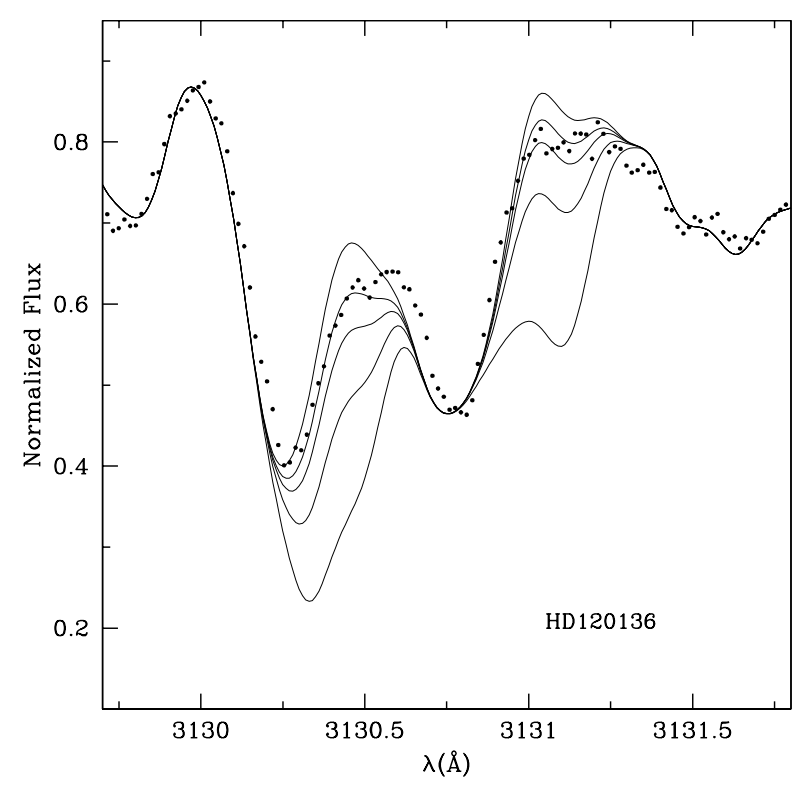

Fig. 3. Spectral syntheses (lines) and observed spectra (dots) in the Be II line region for the star HD 120136 ( $\tau$ Boo). The different curves correspond to syntheses with Be abundances of 1.42 (meteoritic), $0.65,0.25,-0.05$, and -10.0 (no Be). the Be abundance of $\tau$ Boo $(+0.25)$ should be considered as an approximate value.

\subsection{Errors}

Measuring the uncertainties in the determination of Be abundances in not an easy task (we refer to García López et al. 1995a, for a more thorough discussion). In this paper we have adopted the following procedure. First of all, we considered that from the errors of $\pm 50 \mathrm{~K}$ in temperature and $\pm 0.12 \mathrm{dex}$ in $\log g$ we can expect typical uncertainties around 0.03 and 0.05 dex, respectively. An error of 0.05 dex was further added to take into account the fact that there are several $\mathrm{OH}$ lineblends in the Be line region; uncertainties in the oxygen abundance will affect the location of the pseudo-continuum and introduce errors in the final Be abundance.

These values were estimated by fitting the solar spectrum and varying the different parameters. This procedure also revealed that errors in other atmospheric parameters, like the metallicity and the microturbulence (with a derived uncertainty of the order of $0.05 \mathrm{dex}$ and $0.10 \mathrm{~km} \mathrm{~s}^{-1}$, respectively - see Sect.3.1), do not influence the results significantly. 
Our experience also showed that the final Be abundance was slightly sensitive to the values for the broadening parameters used. We have thus added an extra 0.05 dex uncertainty due to errors in the measured spectral resolution, macro-turbulence, and $v \sin i$.

Added quadratically, these figures produce an uncertainty of 0.09 dex, which was added to the error due to continuum placement. This latter was estimated when fitting the spectrum and quantifies the quality of the fit. The final errors are of the order of $0.10-0.15 \mathrm{dex}$; they are usually higher for the cooler dwarfs, and quite small for solar and hotter stars.

García López et al. (1995b) studied in detail the sensitivity of the observed feature at $\lambda 3131 \AA$ to the Be abundance for low-mass stars. For effective temperatures below $\sim 5100 \mathrm{~K}$ the feature starts to be dominated by the contribution of another element (likely Mn I). This makes it difficult, and sometimes impossible, to determine accurate Be abundances for the coolest stars of our sample; the errors involved in the Be determinations are higher for these stars.

The usefulness of the Be II line for deriving Be abundances will thus decrease with temperature to a point where it will be useless. However, the exact $T_{\text {eff }}$ where this will occur will depend on the resolution and signal-to-noise ratio of the observed spectra. And even if the observed feature is not dominated by the $\mathrm{Be}$ transition, it is possible to obtain a reliable Be measurement by fitting synthetic spectra with different Be abundances, as long as the $S / N$ of the data is high enough.

In this sense, three low-mass stars studied (HD 22049 $5073 \mathrm{~K}$, HD $26965 \mathrm{~A}-5126 \mathrm{~K}$, and HD 74576 - $5000 \mathrm{~K}$ ) do have spectra with signal-to-noise ratios high enough to allow precise fitting and an abundance measurement. The analysis of other objects with similar or cooler temperatures just provide upper limits, reflecting both these difficulties and the decrease of Be abundances with decreasing $T_{\text {eff }}$ (see Sect. 5). Examples of fits for these stars can be seen in Figs. 1 and 2: HD 22049 and HD 26965 A, with Be measurements, and HD 13445, with just an upper limit.

\subsection{Instrumental offsets}

Given that our data were obtained with three different instruments, it is important to check for possible systematic errors in the Be abundances obtained from different runs. This is particularly important since, for example, most of the IACUB spectra concern planet-host stars, while most of the UVES(C) data regard comparison sample objects. If the systematic differences between the different instruments are significant, such a result could compromise a comparison between the planet hosts and the comparison sample stars.

Sources of systematic errors can be related, for example, to errors in the background correction during the reduction and extraction of the spectra. If the flux in the spectral region of interest is very low, we might expect a more uncertain background correction. Other errors might arise from the resolution achieved in the different spectra. The IACUB data used in this paper have a spectral resolution close to the limit as imposed by the ability to resolve the Be II features as well as other

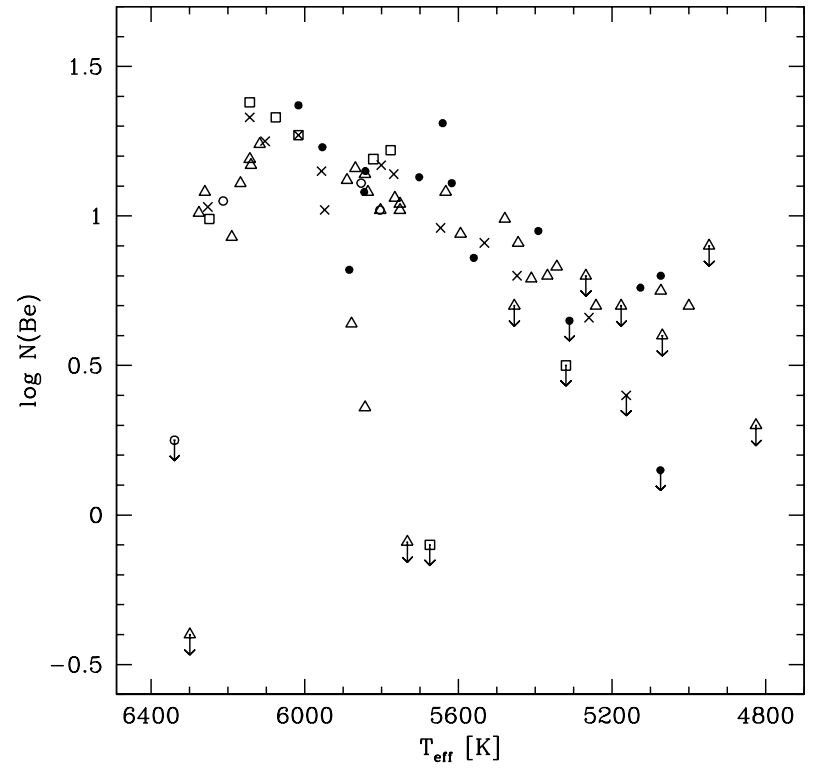

Fig. 4. Derived Be abundances as a function of effective temperature for the stars listed in Tables 2 and 3. Crosses represent results from UVES(A) spectra, open squares from UVES(B), open triangles from UVES(C), open circles from UES, and dots represent Be abundances as derived from IACUB spectra.

lines in the region. As discussed in García López et al. (1995a), for spectra with resolution lower than $\sim 30000$ the Be abundances can no longer be accurately obtained. Furthermore, errors in the smoothing parameters used to fit the spectra (e.g. errors in the estimated spectral resolution itself) might also induce considerable deviations in the final abundances; this is particularly true for the lowest resolution spectra, where the instrumental profile dominates.

In Table 2, three of our target stars (HD 22049, HD 75289, and HD 82943) have more than one Be abundance measurement, obtained using spectra taken with different instruments, or in different observing runs. This gives us the possibility of testing our results for systematic errors due to the different instrumental sets.

In general, the values in the table seem to suggest that no major systematic differences exist. A very small, and not significant difference $(\sim 0.05 \mathrm{dex})$ is found between the $\log N(\mathrm{Be})$ derived from the IACUB and UVES, and no major conclusions can be drawn with the number of comparison points available. Note, however, that the IACUB spectrum obtained for HD 82943 has a particularly low signal-to-noise ratio.

In Fig. 4 we present a plot of Be abundances for our targets as a function of $T_{\text {eff }}$. In the figure, the different symbols indicate different instruments with which the spectra used to derive the Be abundances were obtained. This plot further attests that there are no significant differences between the Be abundances derived from the different instruments.

\section{Li abundances}

The lithium abundances presented in Tables 2 and 3 are revised versions of those derived by Israelian et al. (2004). The new values were obtained using the "new" stellar atmospheric parameters presented in Santos et al. (2004a), the same used to 


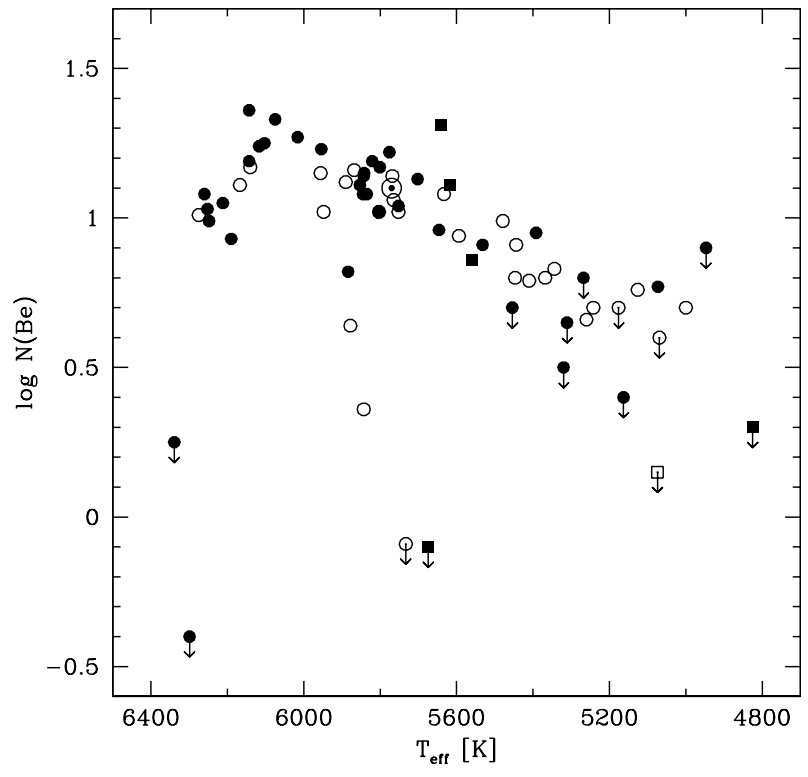

Fig. 5. Derived Be abundances as a function of effective temperature for the stars listed in Tables 2 and 3. Planet hosts are denoted by the filled symbols, while open symbols denote "single" stars. Circles represent dwarfs, while the squares indicate sub-giants. The Sun is denoted by the usual symbol.

derive the Be abundances. The differences with the values listed by Israelian et al. (2004) are always very small, as expected, since the formerly used atmospheric parameters were not very different from the current ones.

For a few stars Israelian et al. (2004) had not derived Li abundances. These were now computed in the same way as in their former work. We refer to this paper for details. In the rest of this paper, the solar Li abundance was taken from Grevesse \& Sauval (1998), $\log N(\mathrm{Li})=1.10$.

\section{Be in planet-host stars}

In Fig. 5 we plot the derived Be abundances as a function of effective temperature for our program stars. In the plot, the open symbols denote "single" stars, while closed symbols represent planet hosts.

In this figure, sub-giants are denoted with squares (see Paper II). The Be abundances for these stars should be taken with caution in the following comparison, since their convective envelopes are deepening. Our sample does not have many stars in this situation, and we prefer not to include these in most of the discussion. The Be abundances of these objects have been further discussed in Paper II.

A visual inspection of Fig. 5 tells us that at a first sight, and overall, no clear difference seems to exist between the two populations of stars. Planet hosts and "single" stars describe the same trend in the $\log N(\mathrm{Be})$ vs. $T_{\text {eff }}$ space. Globally, the Be abundances of both samples decrease from a maximum near $T_{\text {eff }}=6100 \mathrm{~K}$, towards both higher and lower temperature regimes (for a throughout discussion see Paper II). This observation is in agreement with former results on the subject (Santos et al. 2002a), and is similar to the trend observed for $\mathrm{Li}$ (see Fig. 8).

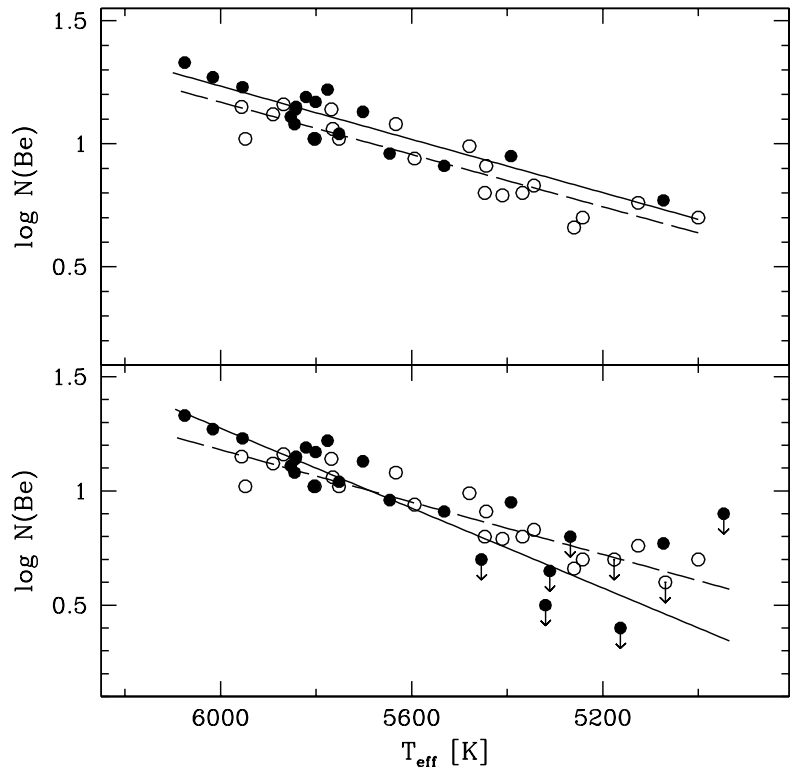

Fig. 6. Be abundances as a function of effective temperature for planet hosts (dots) and comparison sample stars (open circles). Only dwarfs with $T_{\text {eff }}$ below $6100 \mathrm{~K}$ were considered. The 4 solar-temperature dwarfs that fall outside of the main trend were not included wither (see text for more details). Upper panel: The two lines represents linear fits to each of the two groups of stars (continuous line for planet-hosts, and dashed line for comparison sample stars); only objects with Be detections were considered in this plot. Lower panel: Same as above, but when upper limits are also considered. The two lines represent fits to the data using a Buckey-James method (see text for more details).

In Fig. 6 (upper panel) we further plot the Be abundances as a function of effective temperature for planet hosts (dots) and comparison sample dwarfs (open circles) with effective temperatures below $6100 \mathrm{~K}$. Only stars with Be determinations are considered (upper limits were not used). The group of dwarfs with solar temperature that fall considerably below the global trend were also excluded from this plot. As we discuss in Sect. 5.1 and more thoroughly in Paper II, these stars seem to define a Be gap. Two linear fits to the data are also shown, the continuous line denoting a fit to the planethost star points, and the dashed line a fit to the comparison sample star data. As we can see from the figure, and although the visual difference between the two groups of points is not very clear, there seems to be a systematic difference in the sense that planet hosts are more Be-rich than comparison sample stars. This difference, almost constant for all the temperatures, is of the order of 0.05 dex. The slopes of the two fits are of $0.56 \pm 0.07 \mathrm{dex} / 1000 \mathrm{~K}$ (planet-hosts) and $0.53 \pm 0.07 \mathrm{dex} / 1000 \mathrm{~K}$ (comparison stars), and the intercept values are $-2.1 \pm 0.4$ dex (planets) and $-2.0 \pm 0.3 \mathrm{dex}$ (comparison). The rms of the fits is 0.06 and 0.07 for planet hosts and comparison sample stars, respectively.

If we take into account the upper-limit measurements, the situation is a bit different. In Fig. 6 (lower panel) we present such a comparison. The two fits were now done with a Buckey-James method, using ASURV Rev. 1.4 (LaValley et al. 1992), which implements the methods presented in Isobe et al. (1986). The slopes and intercept values (slope, intercept) for 
the two cases are $(0.87 \pm 0.10,-3.97)$ for planet-hosts, and $(0.57 \pm 0.06,-2.26)$ for comparison stars. These fits seem to suggest that for the lowest-temperature stars in our sample, planet hosts may be a bit more Be-poor than comparison sample stars. The difference in the slope of the two samples is mostly due to the few upper-limit Be abundances obtained for the planet hosts in the temperature regime bellow $\sim 5500 \mathrm{~K}$, while for hotter dwarfs the difference is not as large.

In this sense, it has been shown by Israelian et al. (2004) that the $\mathrm{Li}$ abundances of planet-hosts are not above the ones found for field dwarfs. Instead, there is some evidence that they are lower in the temperature regime between 5600 and $5850 \mathrm{~K}$ (see Israelian et al. 2004). This fitting result could be hinting at a similar result for $\mathrm{Be}$ in the lower temperature regime. However, the number of points available is probably not enough to reach a definite conclusion regarding this matter.

Given the low number of measurements, these results do not seem to be particularly significant. All the two-sample tests described in ASURV (see Feigelson \& Nelson 1985) give a low probability that the two samples are not part of the same distribution ${ }^{3}$. Furthermore, it should be mentioned that the Buckey-James method to fit data containing non-detections must be taken with caution, since it assumes that the upper limits in a given experiment are precisely known, while here these somehow represent " $n$ " times the photon noise of the spectra.

On the other hand, if we "believe" in the upper panel of Fig. 6, what could possibly explain the apparent Be-enhancement in planet-host stars?

We can probably exclude that it is due to any pollution effects, since the accretion of hydrogen-poor, metal-rich material would induce a larger variation for the hottest stars in Fig. 6, since these have shallower convective envelopes. This is not seen in the fits (if pollution was responsible for the observed difference, then this would also impose a strict limit on the quantity of accreted material). Curiously, a higher slope is found for planet hosts once we take into account the upper-limit measurements (Fig. 6, lower panel). But as mentioned above, the difference between the two fits (planet hosts and comparison) in this case is mostly due to the lower temperature points, and not to the hotter dwarfs, which would be more sensitive to pollution effects.

One better possibility is that the observed difference is due to galactic evolution effects. Planet hosts are metal-rich when compared with the "single" stars in our sample. As was shown e.g. by Rebolo et al. (1988), Molaro et al. (1997), and Boesgaard et al. (1999), there is a trend for the Be abundances to increase with increasing metallicity, reflecting the Be evolution of the galaxy. A multi-linear fit to the stars in the upper panel of Fig. 6 gives $\log N(\mathrm{Be})=-2.00+$ $0.53\left(T_{\text {eff }} / 1000\right)+0.12[\mathrm{Fe} / \mathrm{H}]$, a relation suggesting that for a given temperature the Be abundances increase with the metallicity (this point will be further discussed in García López et al., in preparation). Since NLTE effects on the Be abundances are not very strong (García López et al. 1995b), they may not be the cause of this difference for stars with different metallicity.

\footnotetext{
3 This is true, whether we fit and subtract the trend of decreasing Be with decreasing temperature or not.
}

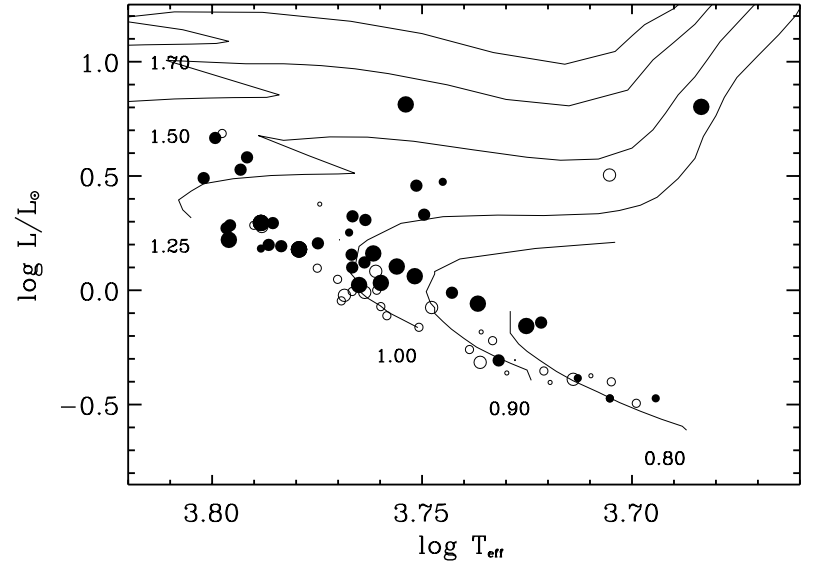

Fig. 7. HR diagram for the stars discussed in the current paper. Open circles denote comparison sample stars, while planet hosts are shown as closed circles. The size of the symbols is proportional to the stellar metallicity. Solar metallicity isochrones from Lejeune \& Schaerer (2001) for 0.8 to $1.7 M_{\odot}$ are also shown.

In Fig. 7 we present an HR-diagram of the stars studied in this paper. The size of the symbols is proportional to the stellar metallicity. The luminosity was computed using Hipparcos parallaxes and $V$ magnitudes (ESA 1997), and the bolometric correction of Flower $(1996)^{4}$. Planet hosts are denoted by open symbols, while comparison sample stars are presented as closed symbols. As can be seen from the plot, planet hosts seem to be, for a given temperature, a bit more luminous than comparison sample stars. This difference may be related to the higher average metallicity of the planet-host stars (e.g. Santos et al. 2004a), or to a slighly different evolutionary status of these stars (radial-velocity surveys for planets are more sensitive to older, non-active stars - Santos et al. 2000). However, this difference also means that planet hosts are, for a given temperature, a bit more massive than comparison sample objects. Whether this mass "excess" could (also) be responsible for the eventual difference observed in Fig. 6 is not clear at this moment.

In general, these results argue against pollution as the key process leading to an overall metallicity excess of stars with planets (see e.g. Santos et al. 2003b, 2004a; Pinsonneault et al. 2001). Even the 0.05 dex difference observed in Fig. 6 (upper panel), if due to any pollution events, would not suffice to explain the difference in the observed $[\mathrm{Fe} / \mathrm{H}]$. For example, adding $\sim 50$ earth masses of $\mathrm{C} 1$ chondrites to the Sun would increase its iron abundance by about $0.25 \mathrm{dex}$ (a value similar to the average difference observed between stars with and without detected giant planets), and its Be abundance would increase by a slightly higher factor ${ }^{5}$. No difference of such magnitude seems present in our data.

\footnotetext{
${ }^{4}$ Since the values in his tables are wrong, we have derived the calibration of $T_{\text {eff }}$ vs. BC by fitting the data in the paper in the same way as he did.

${ }^{5}$ For this calculation we have used a $\mathrm{Be} / \mathrm{Fe}$ ratio similar to $\mathrm{C} 1$ chondrites, and considered an iron content of $\sim 20 \%$ by mass (Anders \& Grevesse 1989).
} 


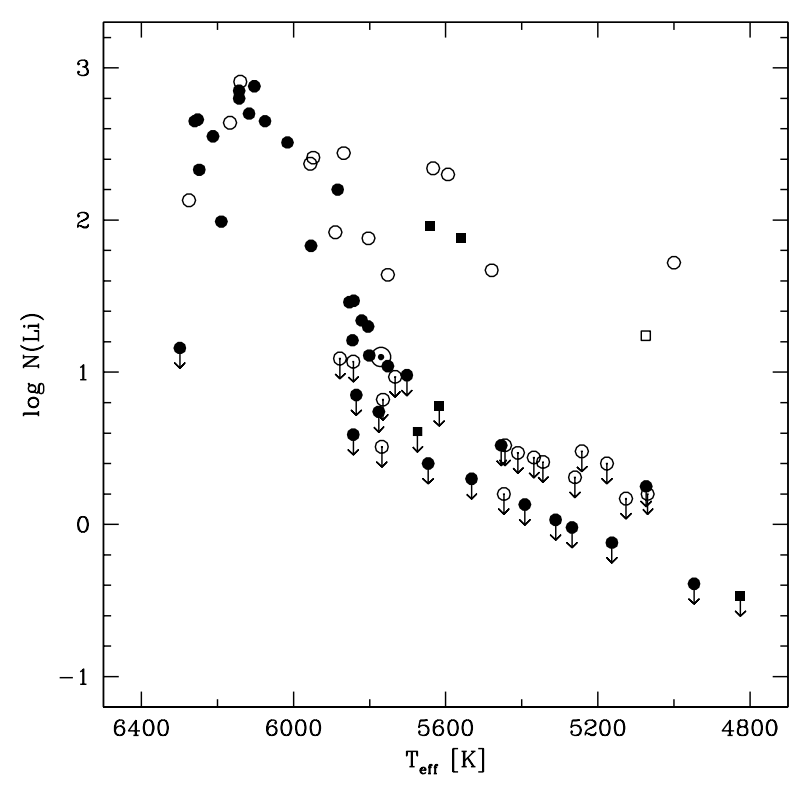

Fig. 8. Li abundances as a function of effective temperature for the same stars as plotted in Fig. 5. The Sun is denoted by the usual symbol.

In the same way, the results do not support either extra mixing due to an eventual different angular momentum history of the two "populations" of stars. As mentioned above, Israelian et al. (2004) (see their Fig. 5) have shown that in the temperature regime between 5600 and $5850 \mathrm{~K}$, planet hosts present lower Li abundances than do single field dwarfs. This difference is not clear for Be (see Figs. 5 and 6), although the result shown in Fig. 6 (lower panel) could hint at this being the case for lower temperature stars.

\subsection{Beryllium anomalies}

A few solar temperature stars in Fig. 5 seem to show particularly low Be abundances. These objects, mostly "single" stars, seem to define a Be-gap for temperatures between roughly 5600 and $6000 \mathrm{~K}$. At this moment we have no reasons to believe that these strange anomalies have anything to do with the presence (or not) of planets. However, more data are needed.

In Santos et al. (2002a) we mentioned that contrary to what is expected from the Be depletion models including rotational mixing (e.g. Pinsonneault et al. 1990), the Be abundances show a decreasing trend as a function of decreasing effective temperature. A few possible explanations for this trend were discussed, including the possibility that it has some relation to the presence of planets around the targets. At that time, however, the "comparison" stars with Be abundances available were completely outnumbered by the planet hosts. The current analysis has now overcome that problem, and seems to show that the same trend is present for both planet-hosts and "single" stars. In other words, the decreasing trend observed in Fig. 5 does not seem to be related to the presence or not of a planet orbiting the star, and is probably related to the Be depletion mechanisms. It should be mentioned, however, that e.g. Boesgaard \& King (2002) and Boesgaard et al. (2003) have not found the same trend in their studies of young open-cluster $F$ and $\mathrm{G}$ dwarfs.

We refer to Paper II for a thorough discussion of these observed anomalies.

\subsection{Lithium and beryllium}

In Fig. 8 we present a plot of the $\mathrm{Li}$ abundances as a function of effective temperature for the stars in our sample. This plot shows the usual behavior of Li with temperature, and with only a few exceptions, no particular difference seems to exist between the two groups of stars (see Israelian et al. 2004). However, 4 objects in Fig. 8 (HD 36435, HD 43162, HD 43834, and HD 74576), all "single" dwarfs, with effective temperatures below $5700 \mathrm{~K}$, have particularly high Li abundances, clearly falling off the main trend. As we discuss in PaperII, however, and except for HD 43162, their position in this plot is probably due to their young ages, and has probably nothing to do with the presence or not of a planet. However, the last mentioned star is particularly troubling, since it is not clear if it is young. If its youth cannot explain the high-Li content of HD 43162, then other explanations have to be considered. Either this star has recently engulfed planetary material (Li-rich and $\mathrm{H}$-poor), thus increasing considerably its Li content, or for some reason it has depleted Li at lower rates than other dwarfs with a similar temperature.

There are also three sub-giants in the above mentioned temperature regime (HD 10697 and HD 117176, both planet hosts, and HD 23249, a "single" star), presenting observable Li abundances. The first two of these have been discussed in Sect. 5.3 as well as in Santos et al. (2002a). All these stars have been further analyzed in Paper II. Amongst these three stars, HD 23249 is the most interesting case, since its Be abundance is clearly depleted, while it still has observable quantities of $\mathrm{Li}$. This result might imply that "pollution" mechanisms have affected the photosphetic composition of this sub-giant.

A comparison of the $\mathrm{Li}$ and $\mathrm{Be}$ abundances for the dwarfs in our sample is presented in Fig. 9. A look at the figure suggests that globally there are no clear differences between planet hosts (closed circles), and "single" stars (open circles). In general, the stars follow the expected trend: stars that are Be depleted are also severely Li depleted. There are, however, a few "single" stars with "high" values of $\mathrm{Li}$ and low values of Be. These cases correspond mostly to the Be-gap stars discussed in Sect. 5.1, and their position in the plot has probably nothing to do with the presence (or not) of planets. In Paper II we discuss these points in more detail.

Curiously, there seems to be a lack of planet hosts with Li abundances $(\log N(\mathrm{Li}))$ between roughly 1.5 and $2.5 \mathrm{dex}$, a region that is populated by "single" stars. All planet hosts in this region have, in general, lower Be abundances than do stars without known planets. We note that this Li gap was also discussed in Israelian et al. (2004). Interestingly, the opposite effect seems to occur for $\mathrm{Li}$ abundances around $\log N(\mathrm{Li})=1.0$, a region for which most of the targets are planet-hosts. We note that at least part of these trends might be the result of the 


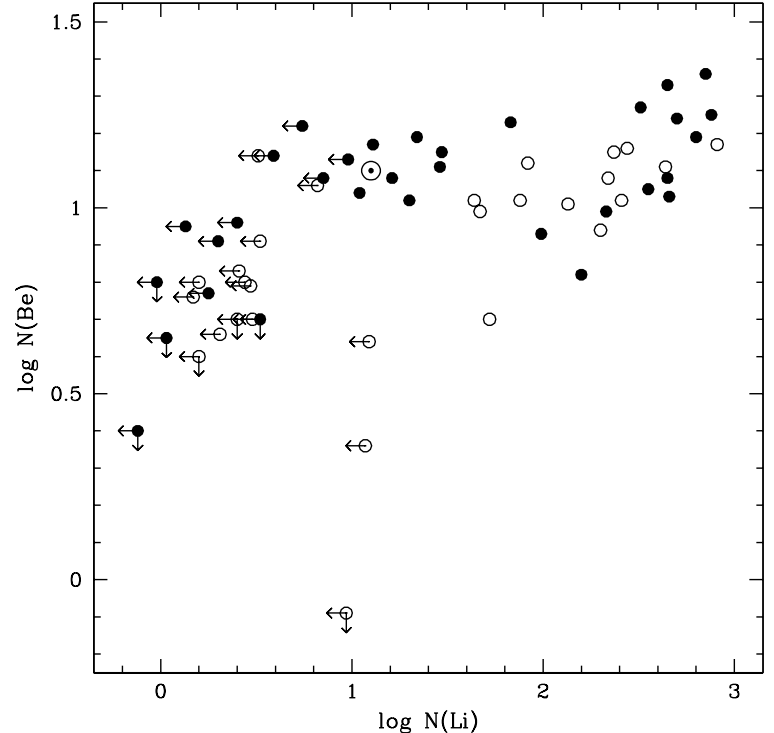

Fig. 9. $\mathrm{Li}$ and Be abundances plotted against each other. Only dwarfs were considered in this plot. Closed circles represent planet hosts, while open symbols denote comparison sample stars. The Sun is denoted by the usual symbol.

non-uniform temperature distribution of our two samples (planet hosts and comparison sample stars).

The small group of planet hosts with high $\mathrm{Li}$ and high Be abundances described in Sect. 5.3 (see below) is also clear in this plot as the small group of points in the upper-right corner of the diagram.

\subsection{Outliers: Hints of "pollution"?}

Interestingly, there is a group of planet-host stars with $T_{\text {eff }}$ between 6000 and $6200 \mathrm{~K}$ that present particularly high Be abundances. All these stars (HD 52265, HD 75289, HD 82943, HD 121504, and HD 209458) have [Fe/H] above solar, in the range from 0.02 for HD 209458 up to 0.30 dex for HD 82943, and Be abundances from 1.24 to 1.36 dex. Given that their shallow convective envelopes are particularly easy to "pollute" (see e.g. discussion in Pinsonneault et al. 2001), this group of stars could represent a sample of objects that have seen their atmospheric abundances altered by the accretion of metal-rich, hydrogen-poor planetary material.

Of this group of objects, HD 82943 is probably the best candidate. In fact, this star has already been found to have an anomalous abundance of ${ }^{6} \mathrm{Li}$, an indication that it might have engulfed a planet, or at least planetary material (Israelian et al. 2001, 2003). The current result might thus confirm the former suspicions.

Unfortunately, the maximum in the Be abundances observed in this temperature range is very narrow, and we do not have many comparison stars in this temperature regime to verify whether this result can be due to the presence of planets or not. Furthermore, on either side of this temperature interval (i.e. for $T_{\text {eff }}>6150$ and $T_{\text {eff }}<6000$ ), planet hosts and comparison sample stars do not show any remarkable difference. This peak in the Be abundances may simply correspond to a region for which Be depletion occurs at lower rate.
Alternatively, and given that these objects are quite metalrich, their initial Be abundances could have been already higher when compared with other stars (Rebolo et al. 1988; Molaro et al. 1997; Boesgaard et al. 1999), thus explaining their position in the plot (see also Sect. 5).

A look at Fig. 8, where we plot the Li abundances as a function of effective temperature for the same stars plotted in Fig. 5 might also give us some information about this point. In fact, these stars do not seem to present particularly high $\mathrm{Li}$ abundances when compared with e.g. the stars without planets that are present in the same temperature regime, although a peak in the $\mathrm{Li}$ abundances also appears at this temperature.

A sixth star may also deserve a comment. HD 10697, a subgiant with a temperature of $5640 \mathrm{~K}$, seems to have a particularly high Be abundance. This case was already discussed in Santos et al. (2002a), as the sub-giant that together with HD 117176 presented a $\mathrm{Li}$ abundance that was high for its temperature. Given the uncertainties in the derived abundances, due e.g. to uncertainties in the surface gravity (see García López \& Pérez de Taoro 1998), and to the use of a relatively lowresolution IACUB spectrum, and given that there may exist non-uniformities in the initial Be content of the stars, it is not clear if this object is exceptionally Be-rich. If so, however, this could mean one of various things: either this is a case where planetary material has been engulfed, or the excess Be is due to a dredge-up effect from a "buffer" below the former main-sequence convective envelope (Deliyannis et al. 1990). It should be mentioned, however, that this latter scenario does not seem to be supported by current observations (e.g. Randich et al. 1999). The cases for the other sub-giants are discussed in Paper II.

\section{Concluding remarks}

In this paper we have obtained $\mathrm{Be}$ abundances for a set of 41 planet-host stars, and a smaller sample of 29 stars not known to harbor any planetary-mass companion. The abundances were derived from a detailed spectroscopic analysis, and gave us the possibility to look for possible differences between the two samples.

A comparison of the Be abundances of planet hosts and "single" stars has revealed that, perhaps with a few exceptions, the two samples follow the same behavior in the $\log N(\mathrm{Be})$ vs. $T_{\text {eff }}$ plot. A small offset of 0.05 dex in Be abundance might be present for all temperatures, planet hosts being more Be-rich. This small difference is tentatively explained as due to the galactic chemical evolution.

The results presented support the idea that the excess metallicity observed for planet hosts has, overall, a "primordial" origin, and is not due to generalized stellar pollution processes. Nevertheless, we have found a small group of stars that present particularly high Be abundances that could be explained by pollution events, although other explanations are possible. More data are needed to allow us to take any conclusion.

In a separate paper (Paper II) we further analyse our results in the framework of the models of Be depletion in solar-type stars. 
Acknowledgements. We wish to thank the Swiss National Science Foundation (Swiss NSF) for the continuous support for this project. Support from Fundação para a Ciência e Tecnologia (Portugal) to N.C.S. in the form of a scholarship is gratefully acknowledged.

\section{References}

Anders, R., \& Grevesse, N. 1989, Geochim. Cosmochim. Acta, 53, 197

Barnes, S., Sofia, S., \& Pinsonneault, M. 2001, ApJ, 548, 1071

Bodaghee, A., Santos, N. C., Israelian, G., \& Mayor, M. 2003, A\&A, 404, 715

Boesgaard, A. M., \& King, J. R. 2002, ApJ, 565, 587

Boesgaard, A. M., Deliyannis, C. P., King, J. R., et al. 1999, AJ, 117, 1549

Boesgaard, A. M., Armengaud, E., \& King, J. R. 2003, ApJ, 582, 410

Chmielewski, Y., Müller, E. A., \& Brault, J. W. 1975, A\&A, 42, 37

Deliyannis, C. P., Demarque, P., \& Kalawer, S. 1990, ApJS, 73, 21

Deliyannis, C. P., Cunha, K., King, J. R., \& Boesgaard, A. M. 2000, AJ, 119, 2437S

Ecuvillon, A., Israelian, G., Santos, N. C., et al. 2004, A\&A, 418, 703

Edwards, S., Strom, S. E., Hartigan, P., et al. 1993, AJ, 106, 372

ESA 1997, The Hipparcos and Tycho catalogue, ESA-SP 1200

Feigelson, E. D., \& Nelson, P. I. 1985, ApJ, 293, 192

Flower, P. J. 1996, ApJ, 469, 355

García López, R. J., \& Pérez de Taoro, M. R. 1998, A\&A, 334, 599

García López, R. J., Severino, G., \& Gomez, M. T. 1995a, A\&A, 297, 787

García López, R. J., Rebolo, R., \& Perez de Taoro, M. R. 1995b, A\&A, 302,184

Gonzalez, G. 1997, MNRAS, 285, 403

Gonzalez, G. 1998, A\&A, 334, 221

Gonzalez, G., \& Laws, C. 2000, ApJ, 119, 390

Gonzalez, G., Laws, C., Tyagi, S., \& Reddy, B. E. 2001, AJ, 121, 432

Goodman, J., \& Rafikov, R. R. 2001, ApJ, 552, 793

Gray, D. 1992, in The observation and analysis of stellar photospheres (Cambridge Univ. Press)

Grevesse, N., \& Sauval, A. J. 1998, Space Sci. Rev., 85, 161

Hartmann, L. 2002, ApJ, 566, L29

Isobe, T., Feigelson, E. D., \& Nelson, P. I. 1986, ApJ, 306, 490

Israelian, G. 2003, in Stars as Suns: Activity, Evolution, and Planets, ed. A. K. Dupree (San Francisco: ASP), IAUS, 219, in press [arXiv:astro-ph/0310377]

Israelian, G., Santos, N. C., Mayor, M., \& Rebolo, R. 2001, Nature, 411, 163

Israelian, G., Santos, N. C., Mayor, M., \& Rebolo, R. 2003, A\&A, 405,753

Israelian, G., Santos, N. C., Mayor, M., \& Rebolo, R. 2004, A\&A, 414,601

Kurucz, R. L. 1993, CD-ROMs, ATLAS9 Stellar Atmospheres Programs and $2 \mathrm{~km} \mathrm{~s}^{-1}$ Grid (Cambridge: Smithsonian Astrophys. Obs.)
Kurucz, R. L., Furenlid, I., Brault, J., \& Testerman, L. 1984, Solar Flux Atlas from 296 to $1300 \mathrm{~nm}$, NOAO Atlas No. 1

Molaro, P., Bonifacio, P., Castelli, F., \& Pasquini, L. 1997, A\&A, 319, 593

LaValley, M., Isobe, T., \& Feigelson, E. D. 1992, BAAS, Rev., 1.1

Laughlin, G., \& Adams, F. C. 1997, ApJ, 491, L51

Laws, C., \& Gonzalez, G. 2001, ApJ, 553, 405

Laws, C., Gonzalez, G., Walker, K. M., et al. 2003, AJ, 125, 2664

Lejeune, T., \& Schaerer, D. 2001, A\&A, 366, 538

Murray, N., \& Chaboyer, B. 2002, ApJ, 566, 442

Naef, D., Mayor, M., Beuzit, J. L., Perrier, C., Queloz, D., et al. 2004, A\&A, 414, 351

Pinsonneault, M. H., DePoy, D. L., \& Coffee, M. 2001, ApJ, 556, L59

Pinsonneault, M. H., Kawaler, S. D., \& Demarque, P. 1990, ApJS, 74, 501

Randich, S., Gratton, R., Pallavicini, R., Pasquini, L., \& Carretta, E. 1999, A\&A, 348, 487

Rebolo, R., Molaro, P., Abia, C., \& Beckman, J. E. 1988, A\&A, 193 , 193

Rebull, L. M. 2001, AJ, 121, 1676

Rebull, L. M., Wolff, S. C., Strom, S. E., \& Makidon, R. B. 2002, AJ, 124, 546

Reddy, B., Lambert, D., Laws, C., Gonzalez, G., \& Covey, K. 2003, MNRAS, 335, 1005

Reid, I. N. 2002, PASP, 114, 306

Ryan, S. 2000, MNRAS, 316, L35

Sadakane, K., Ohkubo, M., Takeda, Y., et al. 2002, PASJ, 54, 911

Santos, N. C., Israelian, G., \& Mayor, M. 2000, A\&A, 363, 228

Santos, N. C., Israelian, G., \& Mayor, M. 2001, A\&A, 373, 1019

Santos, N. C., García López, R. J., Israelian, G., et al. 2002a, A\&A, 386,1028

Santos, N. C., Mayor, M., Naef, D., et al. 2002b, A\&A, 392, 215

Santos, N. C., Mayor, M., Udry, S., et al. 2003a, in Stars as Suns: Activity, Evolution, and Planets, ed. A. K. Dupree (San Francisco: ASP), IAUS, 219, in press

Santos, N. C., Israelian, G., Mayor, M., Rebolo, R., \& Udry, S. 2003b, A\&A, 398, 363

Santos, N. C., Israelian, G., \& Mayor, M. 2004a, A\&A, 415, 1153

Santos, N. C., Israelian, G., Randich, S., García López, R. J., \& Rebolo, R. 2004b, A\&A, 425, 1013 (Paper II)

Sari, R., \& Goldreich, P. 2004, ApJL, 606, 77

Siess, L., \& Livio, M. 1999, MNRAS, 308, 1133

Smith, V. V., Cunha, K., \& Lazzaro, D. 2001, AJ, 121, 3207

Sneden, C. 1973, Ph.D. Thesis, University of Texas

Soderblom, D. R. 1982, ApJ, 263, 269

Stassun, K. G., Mathieu, R. D., Mazeh, T., \& Vrba, F. J. 1999, AJ, 117, 2941

Stephens, A., Boesgaard, A. M., King, J. R., \& Deliyannis, C. P. 1997, ApJ, 491, 339

Strom, S. E. 1994, in 8th workshop on Cool Stars, Stellar Systems, and the Sun, ed. J.-P. Caillault, ASP Conf. Ser., 64, 211

Vauclair, S. 2004, ApJ, 605, 874

Wolff, S. C., Strom, S. E., \& Hillenbrand, L. A. 2004, ApJ, 601, 979 